\title{
Urinary excretion of glycosaminoglycans and hydroxyproline in Paget's disease of bone, compared with neoplastic invasion of bone
}

\author{
LYNNE BOWER, GERALD MANLEY \\ From the Department of Chemical Pathology, Torbay Hospital, Torquay, Devon
}

SUMMARY Urinary glycosaminoglycan and hydroxyproline excretion was studied in 11 patients with clear evidence of Paget's disease of bone. Urinary hydroxyproline, cetyl pyridinium chloride (CPC)precipitable uronic acid and CPC-precipitable hexosamine were expressed as ratios to urinary creatinine. Urine samples were concentrated $\times 1000$ by vacuum dialysis and the glycosaminoglycans examined by electrophoresis on cellulose acetate followed by staining with alcian blue. All the cases studied showed markedly raised hydroxyproline excretion, whereas the uronic acid excretion was normal or only slightly raised in 10 of the 11 cases studied. One patient who had a raised uronic acid and raised hydroxyproline concentration was shown to have osteosarcoma as a complication of Paget's disease.

The very high hydroxyproline:creatinine ratio in all cases of Paget's disease (mean $241.8 \mathrm{mmol}$ hydroxyproline/mol creatinine) contrasted sharply with the cases of disseminated neoplasm, where the ratio was either normal or slightly raised (mean $29.3 \mathrm{mmol}$ hydroxyproline/mol creatinine). The ratio of hydroxyproline to CPC-precipitable uronic acid was also markedly raised in cases of Paget's disease (mean $77.3 \mathrm{mmol}$ hydroxyproline/mmol uronic acid) and was lower in the neoplastic group (mean $14.1 \mathrm{mmol}$ hydroxyproline/mmol uronic acid) but showed no advantage over the hydroxyproline: creatinine ratio in differentiating the two groups.

The urinary hydroxyproline:creatinine ratio promises to be of value in differentiating between Paget's disease of bone and neoplastic invasion of bone. A marked rise in CPC-precipitable uronic acid excretion alone is more suggestive of neoplastic invasion of bone, and if associated with a marked increase in hydroxyproline excretion, it raises the possibility of neoplastic change in Paget's disease of bone. The results of this study also suggest that bone collagen, rather than bone tissue in general, is primarily affected in Paget's disease.

Increased urinary excretion of hydroxyproline has been reported in cases of Paget's disease of bone. ${ }^{1}$ Hydroxyproline is an imino acid which occurs mainly in fibrous proteins such as collagen. The majority of total body collagen is found in bone. In Paget's disease, where high hydroxyproline excretion is found in conjunction with bone lesions, it seems likely that the source of urinary hydroxyproline is bone collagen.

Glycosaminoglycans have been shown to be associated with collagen in bone. ${ }^{2}$ Urinary excretion of glycosaminoglycans can be studied quantitatively by precipitation with cetyl pyridinium chloride (CPC) followed by measurement of the uronic acid

Accepted for publication 23 March 1981 and hexosamine content of the CPC-precipitable material. Urinary glycosaminoglycans may also be concentrated by vacuum dialysis and separated by electrophoresis. ${ }^{3} 4$ It was decided to study a series of patients with clear evidence of Paget's disease to determine the excretion of hydroxyproline and glycosaminoglycans. The results from these patients were compared with those found in a group of patients with disseminated neoplasm on whom similar studies had been carried out. ${ }^{3}$

\section{Material and methods}

Venous blood $(20 \mathrm{ml})$ and a 24-hour urine collection was taken from 11 patients with radiologically-proven active Paget's disease. The extent of skeletal activity 
was assessed biochemically by serum alkaline phosphatase, calcium, inorganic phosphate and 5-nucleotidase together with 24-hour urinary hydroxyproline excretion. Twenty-four hour urinary glycosaminoglycan excretion was studied by uronic acid assay after isolation by cetyl pyridinium chloride (CPC) precipitation, and electrophoretic separation of urinary glycosaminoglycans concentrated by vacuum dialysis. Control data for glycosaminoglycan excretion was drawn from 100 normal subjects of all ages studied by identical methods and reported previously. 4

\section{Serum alkaline phosphatase}

The method of Morgenstern et al..$^{5}$ was employed, using a Technicon SMA-plus analyser.

\section{Serum calcium}

A method based on the work of Kessler ${ }^{6}$ and Gitelman $^{7}$ was employed, using a Technicon SMAplus analyser.

\section{Serum inorganic phosphate}

A method based on the work of Hurst ${ }^{8}$ and $\mathrm{Kraml}^{9}$ was employed, using a Technicon SMA-plus analyser.

\section{Serum 5-nucleotidase}

This was assayed using the method of Campbell.10

\section{Urine creatinine}

A single-channel Technicon Auto-Analyzer was used (AA1) employing method N-116 (alkaline picrate).

\section{Hydroxyproline assay}

Aliquots of 24-hour urine collections were assayed using the Hypronosticon kit (Organon (Teknika) Ltd, Cromwell Road, St Neots, Huntingdon) which is based on the method of Goverde and Veenkamp. ${ }^{11}$

\section{Isolation of glycosaminoglycans}

Glycosaminoglycans were isolated from aliquots ( $5 \mathrm{ml}$ ) of 24-hour urine samples by CPC precipitation, followed by dissolution of the complex in sodium chloride and precipitation in ethanol. ${ }^{4}$

\section{Uronic acid assay}

Glycosaminoglycans isolated from aliquots of urine $(5 \mathrm{ml})$ were dissolved in water $(1 \mathrm{ml})$ and the uronic acid content was determined using the method of Bitter and Muir. ${ }^{12}$

\section{Hexosamine assay}

Glycosaminoglycans isolated from aliquots of urine $(5 \mathrm{ml})$ were hydrolysed with $\mathrm{HCl}(4 M)$ in sealed glass ampoules in an autoclave at 15 psi for $30 \mathrm{~min} .{ }^{13}$ Hydrolysates were dried over $\mathrm{KOH}$ in vacuo, and hexosamine was assayed by Boas'14 modification of the Elson-Morgan reaction, as previously described. ${ }^{15}$

\section{Separation of glycosaminoglycans}

Aliquots $(50 \mathrm{ml})$ of 24-hour urine samples were concentrated $\times 1000$ by vacuum dialysis. Electrophoresis of urine concentrates $(10 \mu \mathrm{l}$, equivalent to $10 \mathrm{ml}$ original urine) was carried out on cellulose acetate membranes in veronal-acetate buffer, $\mathrm{pH} 9.28$ Marker samples of hyaluronic acid (human umbilicas cord), heparan sulphate (human aorta), and chone droitin sulphate (human aorta) were run alongside urine concentrates. The glycosaminoglycans were located by staining with alcian blue and quantified by reflectance densitometry using the Joyce Chromoscan, as previously described. ${ }^{15}$

\section{Results}

The major results of this study are shown in the Table. All the cases studied showed very high serum

Biochemical data on 11 cases of Paget's disease

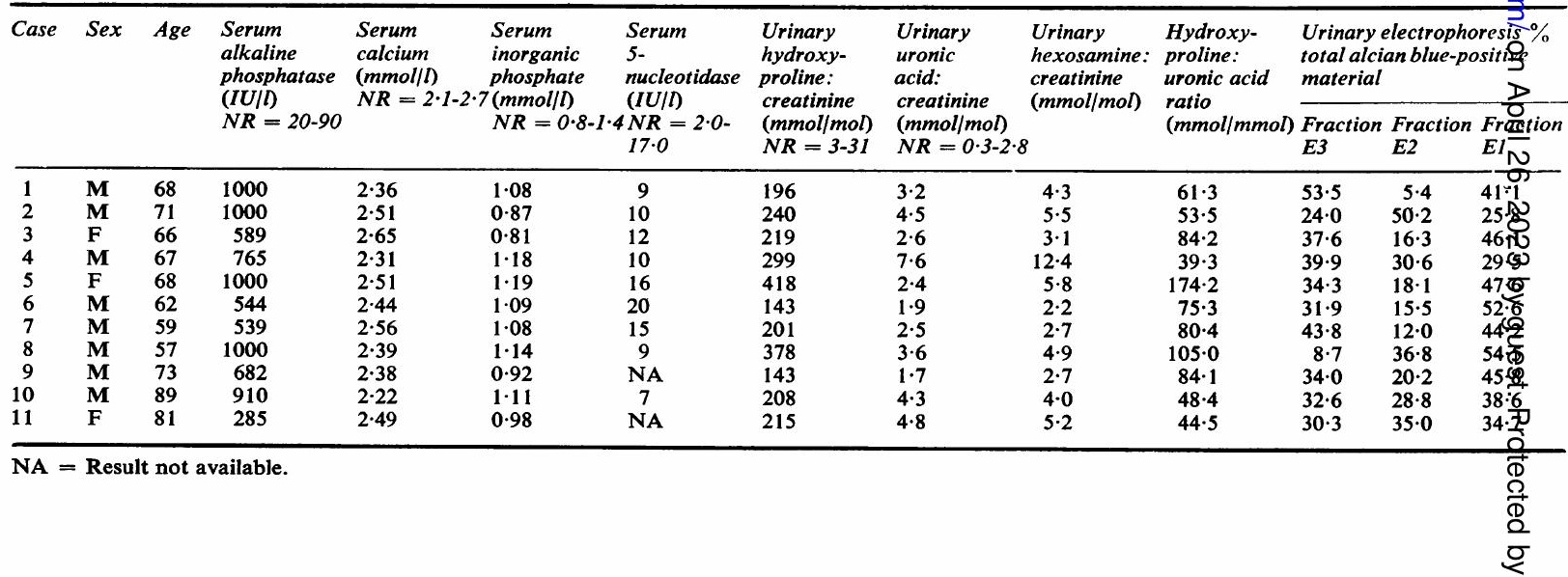


alkaline phosphatase activities. The 5-nucleotidase activities were assayed in nine of the 11 cases, eight of these were within normal limits while one was very slightly raised. The urinary hydroxyproline results were markedly raised in every case. The uronic acid results showed five cases within normal limits, five cases with slightly raised concentrations and only one case (No 4) with a significantly raised uronic acid. The urinary hexosamine:creatinine ratio was higher than the uronic acid:creatinine ratio in all but one case.

A graph of the hydroxyproline and uronic acid results for the cases of Paget's disease and from 24 cases of disseminated neoplasm, previously published, ${ }^{3}$ are shown in Fig. 1. This shows that the pattern of excretion found in Paget's disease is markedly different from that found in disseminated neoplasm.

The urinary hydroxyproline:creatinine ratio was very high in all cases of Paget's disease, ranging from 143 to $418 \mathrm{mmol}$ hydroxyproline/mol creatinine with a mean value of $241 \cdot 8$, and normal or only slightly

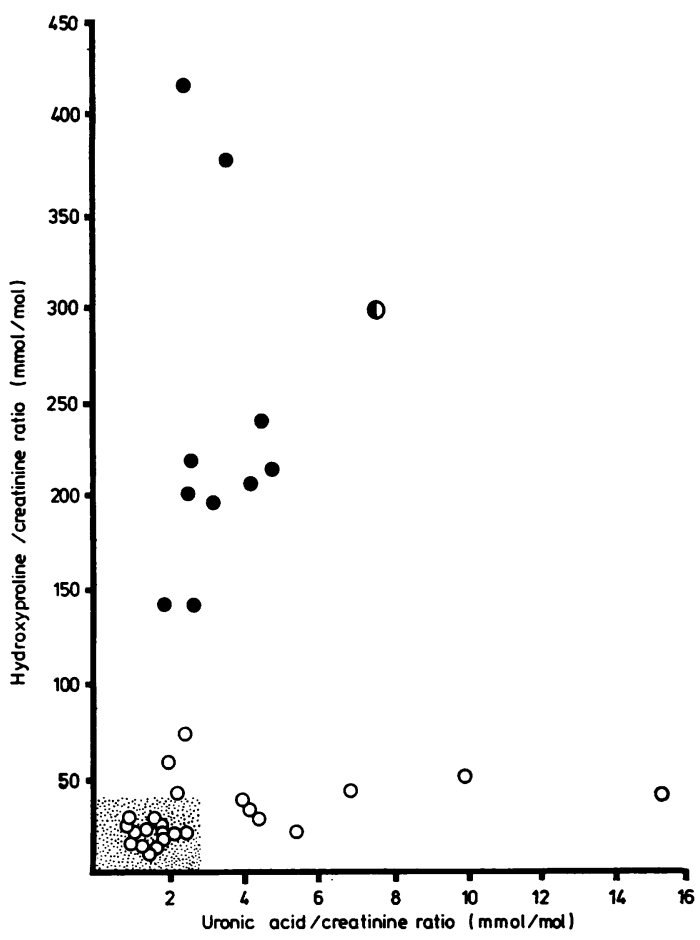

Fig. 1 Urinary hydroxyproline: creatinine and CPC-precipitable uronic acid:creatinine ratios in: 10 cases of uncomplicated Paget's disease of bone, D 1 case of Paget's disease of bone with osteosarcoma and $\bigcirc 24$ cases of disseminated neoplasm. ${ }^{3}$ The stippled rectangle represents the normal range. raised in cases of disseminated neoplasm, where the range was 10 to $74 \mathrm{mmol}$ hydroxyproline/mol creatinine, with a mean value of $29.3 \mathrm{mmol}$ hydroxyproline/mol creatinine.

The ratio of hydroxyproline to CPC-precipitable uronic acid was also greatly raised in cases of Paget's disease (range 39.3-174.2 mmol hydroxyproline/mmol uronic acid, mean $77 \cdot 3$ ) compared with cases of disseminated neoplasm, where the range was $2 \cdot 7-34.8 \mathrm{mmol}$ hydroxyproline $/ \mathrm{mmol}$ uronic acid, mean $14 \cdot 1$. The urinary hydroxyproline: creatinine ratio in cases of uncomplicated Paget's disease compared with cases of disseminated neoplasm with clear radiological evidence of skeletal metastases is shown in Fig. 2, and the urinary hydroxyproline: uronic acid ratio is shown in Fig. 3.

Electrophoresis of urine concentrates showed three alcian blue-positive fractions in each case, as described previously. ${ }^{4}$ The fractions are identified as E1, E2 and E3 where E1 corresponds in electrophoretic mobility and hyaluronidase lability to chondroitin sulphates and dermatan sulphate, E2 corresponds to heparan sulphate and E3 to the glycoproteins. The proportions of these three

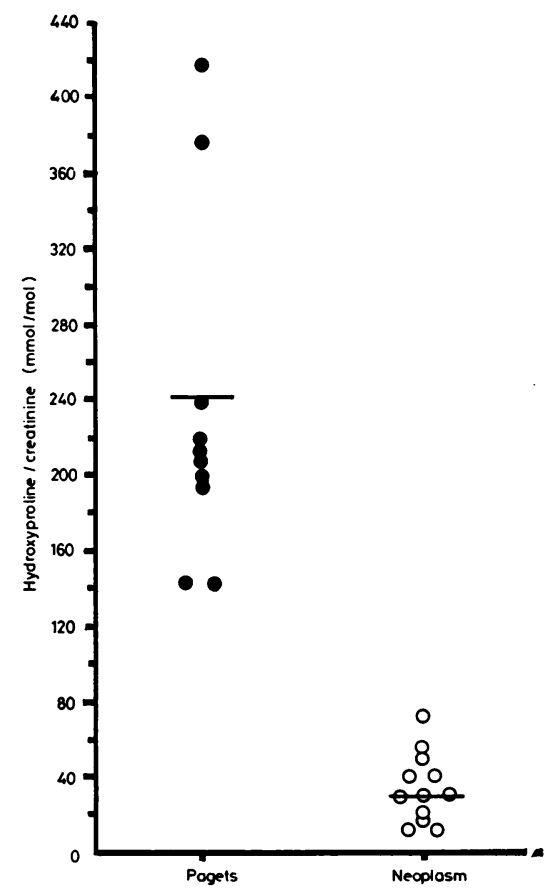

Fig. 2 Urinary hydroxyproline: creatinine ratios in 10 cases of uncomplicated Paget's disease of bone and $\bigcirc 12$ cases of disseminated neoplasm with clear radiological evidence of bone secondaries. ${ }^{3}$ 


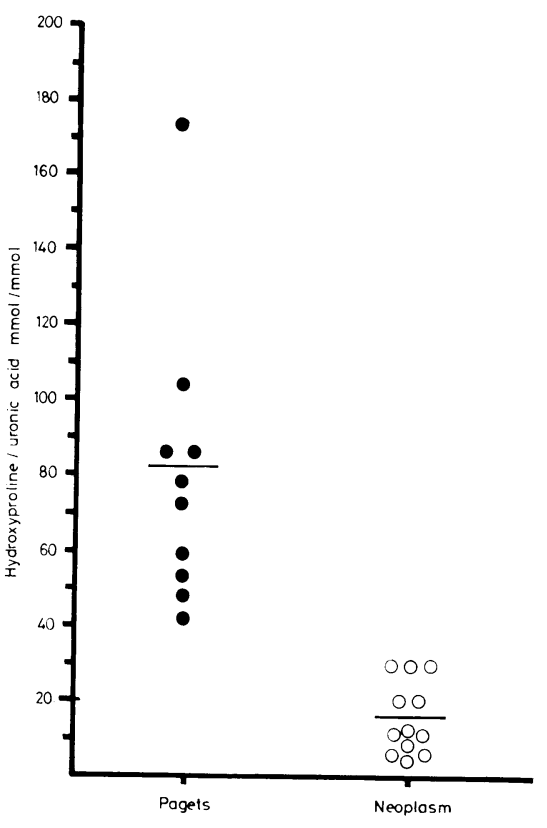

Fig. 3 Urinary hydroxyproline:CPC-precipitable uronic acid ratios in 10 cases of uncomplicated Paget's disease of bone and 12 cases of disseminated neoplasm with clear radiological evidence of bone secondaries. ${ }^{3}$

fractions found in each case are shown as percentages of total alcian blue-positive material in the Table. No abnormal fractions were seen in any of the electrophoretic patterns studied in this series, in contrast to the previously reported series of cases with disseminated neoplasm, where a fraction corresponding to nyaluronic acid was observed in over $50 \%$ of the cases with clear evidence of skeletal involvement.

\section{Discussion}

The object of this study was to ascertain whether there was an increase in urinary glycosaminoglycan excretion as well as urinary hydroxyproline excretion in Paget's disease. The results obtained indicate that half the cases have normal glycosaminoglycan excretion and the other half have only slightly raised excretion. These results contrast with the very high concentrations of urinary hydroxyproline excretion. All the cases studied showed the typical serum biochemistry of Paget's disease; that of normal calcium and inorganic phosphate values together with high alkaline phosphatase values and normal 5-nucleotidase activities.

The urinary excretion of hydroxyproline and uronic acid, illustrated in Fig. 1, show interesting differences between the cases of Paget's disease and $\underline{0}$ the cases of disseminated neoplasm. The majority of

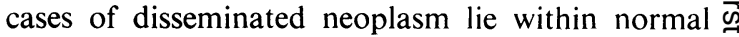
limits for both parameters. For those that stay beyond the normal limits, the trend is clearly to an increase in uronic acid excretion, with only three cases showing a significant increase in hydroxy- $\stackrel{\mathbb{D}}{\circ}$ proline excretion. The pattern in Paget's disease is quite different. None of the cases lies within the normal range for both parameters, but the trend is clearly to a marked increase in hydroxyproline excretion. Only one case of Paget's disease showed a significantly raised uronic acid excretion, and this case (No 4) was found to have developed an osteo- $\omega$ sarcoma in an area of bone previously affected by Paget's disease, and so could be placed in either the Paget's or the neoplastic group.

The urinary CPC-precipitable hexosamine was slightly greater than the CPC-precipitable uronic 을 acid in 10 of the 11 cases. The average molar ratio of hexosamine to uronic acid was $1 \cdot 36$, similar to that found in cases of disseminated neoplasm. The slight excess of hexosamine to uronic acid is presumably due to the presence of keratan sulphates in the CPC precipitate.

The differentiation between Paget's disease of bone and neoplastic infiltration of bone is sometimes vero difficult indeed, even on radiological grounds. In this study, either the urinary hydroxyproline:creatinine ratio, or the urinary hydroxyproline:uronic acid ratio provided a clear differentiation between the two groups. The hydroxyproline:creatinine ratio is easier to measure, but the additional measurement of urinary CPC-precipitable uronic acid excretion may provide useful information in some cases. Thus a marked increase in uronic acid excretion in the presence of a normal or only slightly raised hydroxyproline excretion is more suggestive of neoplastic invasion than Paget's disease of bone, and a marked increase in both hydroxyproline and uronic acid excretion may suggest the possibility of coexistent Paget's disease and neoplastic invasion of bone. The possibility that a rising urinary uronic acid excretion may serve as an early warning of sarcomatous change in Paget's disease of bone deserves further study.

The origin of the increased CPC-precipitable uronic acid in the urine of many cases of disseminated neoplasm is not yet clear, but it seems likely that it arises from degradation of glycosaminoglycans in the ground substance of bone and other connective tissues. ${ }^{3}$ The results of this study of patients with Paget's disease, where a massive increase in urinary hydroxyproline excretion was accompanied by either no increase, or a relatively insignificant increase, in the urinary excretion of CPC-precipitable 
uronic acid, adds further support to the suggestion that bone collagen, rather than bone tissue in general, is primarily affected in Paget's disease. The pattern of hydroxyproline peptides excreted in Paget's disease has been shown to be different from that of normal subjects. ${ }^{16}$ Isotopic studies of the hydroxyproline containing peptides in the urine of patients with Paget's disease have shown that these are related to collagen synthesis. ${ }^{17}$ However, the nature of any defect of collagen synthesis in Paget's disease is not clear at present.

The conclusion to be drawn from this study is that considerable increase in the urinary excretion of hydroxyproline in Paget's disease is not associated with a similar increase in the urinary excretion of CPC-precipitable uronic acid. The urinary hydroxyproline:creatinine or hydroxyproline:uronic acid ratios offer a means of differentiating Paget's disease of bone from neoplastic infiltration of bone on biochemical grounds.

\section{References}

${ }^{1}$ Dull TA, Henneman PH. Urinary hydroxyproline as an index of collagen turnover in bone. $N$ Engl J Med 1963; 268:132-4.

${ }^{2}$ Herring GM, Kent PW. Some studies on mucosubstances of bovine cortical bone. Biochem J 1963;89:405-14.

${ }^{3}$ Manley G, Bower L, Anson A. Urinary excretion of glycosaminoglycans in disseminated neoplasm. J Clin Pathol 1978;31:447-53.

4 Manley G, Severn M, Hawksworth J. Excretion patterns of glycosaminoglycans and glycoproteins in normal human urine. J Clin Pathol 1968;21:339-45.

${ }^{5}$ Morgenstern S, Kessler G, Auerbach J, Flor RV, Klein B.
An automated $p$-nitrophenylphosphate serum alkaline phosphatase procedure for the Auto-Analyzer. Clin Chem 1965;11:876-88.

${ }^{6}$ Kessler G, Wolfman M. An automated procedure for the determination of calcium and phosphorus. Clin Chem 1964;10:686-703.

${ }^{7}$ Gitelman HJ. An improved automated procedure for the determination of calcium in biochemical specimens. Anal Biochem 1967;18:521-31.

${ }^{8}$ Hurst RO. The determination of nucleotide phosphorus with stannous chloride hydrazine sulphate reagent. Can J Biochem 1964;42:287-92.

${ }^{9}$ Kraml M. Clin Chim Acta 1966;13:422.

${ }^{10}$ Campbell DM. Determination of 5-nucleotidase in blood serum Biochem $J$ 1962;82:34.

${ }^{11}$ Goverde BC, Veenkamp FJN. Routine assay of total urinary hydroxyproline based on resin catalysed hydrolysis. Clin Chim Acta 1972;41:29-40.

12 Bitter T, Muir H. A modified uronic acid carbazole reaction. Anal Biochem 1962;4:330-4.

${ }^{13}$ Sobocinski PZ, Canterbury WJ, Jurgens KH. Improved continuous-flow method for determination of total serum bexosamines. Clin Chem 1976;22:1394-6.

${ }^{14}$ Boas NF. Method for the determination of hexosamines in tissues. J Biol Chem 1953;203:553-63.

${ }^{15}$ Manley G, Williams U. Urinary excretion of glycosaminoglycans in the various forms of gargoylism. J Clin Pathol 1969;22:67-75.

16 Dakkak R, Goussault Y, Dreux C. Modification of peptide hydroxyprolinuria during Paget's disease and osteoma. Ann Biol Clin (Paris) 1979;37,4:195-200.

${ }^{17}$ Krane SM, Munoz AJ, Harris ED. Urinary polypeptides related to collagen synthesis. J Clin Invest 1970;49:71629.

Requests for reprints to: Mrs Lynne Bower, Department of Chemical Pathology, Torbay Hospital, Torquay, Devon, England. 